\title{
The importance of citrates in the treatment and prophylaxis of calcium oxalate urinary stones
}

\author{
Michele Barbera $^{1}$, Andreas Tsirgiotis ${ }^{1}$, Mauro Barbera ${ }^{2}$, Quintino Paola ${ }^{1}$ \\ ${ }^{1}$ Operative Unit of Urology, Ospedale Giovanni Paolo II, Sciacca, Italy; \\ ${ }^{2}$ University of Palermo, Palermo, Italy.
}

\begin{abstract}
Summary About $10 \%$ of the people is the subject of an episode of kidney stones during their lifetime, about $70 \%$ of these people undergoes relapses. About $80 \%$ of the urinary stones contains calcium, of wich $80 \%$ is formed of calcium oxalate, in pure form or associated with calcium phosphate. Therefore we can saythat in most cases (about $65 \%$ ) the urinary stones are composedof calcium oxalate.

Use of supplements of potassium citrate and magnesium citrate can help in the prevention of kidney stones of calcium oxalate, but mostly they can be used in the days before a shockwaves lithotripsy treatment to make the stones more fragile to the effect of the shock waves. A case of successful treatment with magnesium potassium citrate of a SWL resistant ureteral stone is presented.
\end{abstract}

KEY WORDS: Calcium oxalate stones prophylaxis.

Submitted 15 November 2016; Accepted 15 December 2016

\section{INTRODUCTION}

Kidney stones are very common in the population, they affect about 3\% of people on average. Particularly at risk are males aged between twenty and forty years. In this age group, because of the simultaneous presence of multiple risk factors, the incidence of the disease is over $15 \%$.

The causes of the origin of the stones have not yet been fully clarified, although some predisposing factors significantly increase the likelihood the formation of the stones:

- male gender: males have a 3 fold increased risk than women to develop kidney stones in the urinary tract (the highest concentration of citrate in women's urine, closely related to the estrogen rate, would explain the lower incidence of the disease in the females);

- poor fluid intake: a reduced flow of urine favors the stagnation of the urine and then the precipitation of the salts contained in the urine as in the case of dehydration due to the increase of fluids loss (diarrhea, sweating);

- age: kidney stones are formed mainly between twenty and forty years;

- acidity of urine: urinary $\mathrm{pH}$ below 5 (with respect to some types of stones, such as uric acid, cystine, and xanthine);
- family history of kidney stones: is the case for example of cystine stones, due to a congenital defect of the kidney which cause high concentration of a poorly soluble amino acid in the urine (cystine) which precipitates forming crystals;

- chronic urinary tract infections;

- abuse of certain drugs or saline and vitamin supplements;

- hyperthyroidism (catabolic effect on bone) and hyperparathyroidism (increased blood calcium);

- incongruous diet;

- ethnicity: increased incidence of kidney stones in white and Asian race;

- climate (during the hot summer period the increased of evaporation, if not supplemented by appropriate fluid intake, increases urine concentration and the formation of stones).

\section{Case report}

A man of 28 years was diagnosed with a $15 \mathrm{~mm}$ radiopaque stones located in the first tract of the left lumbar ureter (Figure 1).

The patient was subjected to a shockwaves litothripsy (SWL) (2) treatment that did not achieve fragmentation of the stone (3).

After that, he underwent left ureterolithotripsy with limited fragmentation of the stone and migration of the frag-

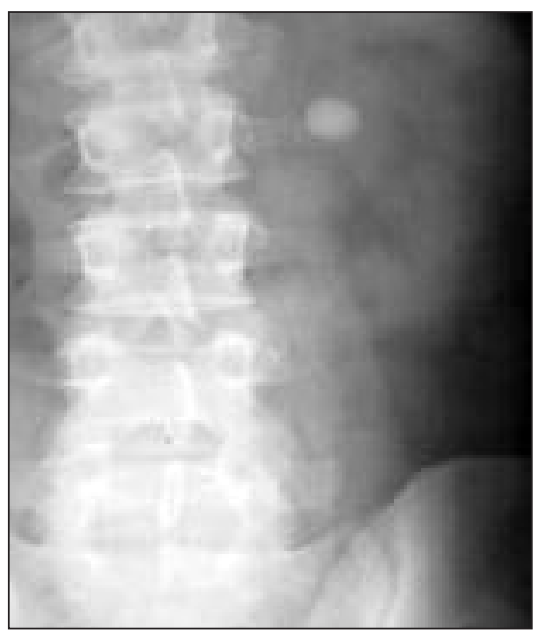

Figure 1.

No conflict of interest declared. 


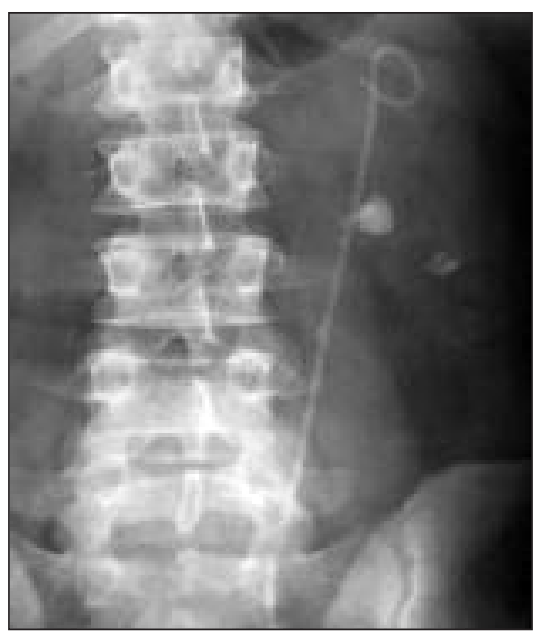

Figure 2.

ments in the lower pole of the left kidney (Figure 2). A left ureteral stent was placed.

He started oral therapy with magnesium and potassium citrate for 30 days as pre-treatment for subsequent SWL (1). After the second SWL treatment fragmentation of the stones in small concretions more evident at the lower calyceal group level was achieved (Figure 3).

The patient continued the treatment with oral magnesium potassium citrate and hydratation. A further reduction of the size of the fragments and the spontaneous expulsion of most of them was observed (Figure 4).

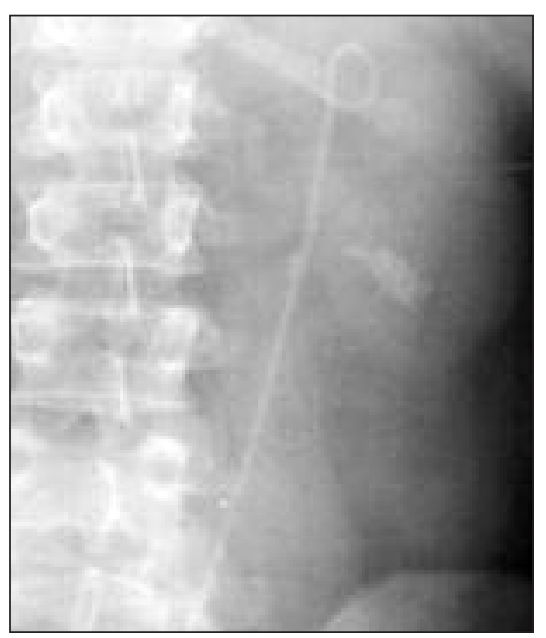

Figure 3.

\section{Correspondence}

Michele Barbera, MD

barbera.mic@gmail.com

Andreas Tsirgiotis, MD

atsirgiotis@alice.it

Paola Quintino, MD

q.paola@inwind.it

Operative Unit of Urology, Ospedale Giovanni Paolo II, Sciacca, Italy

Mauro Barbera, MB

barbera.mau@gmail.com

University of Palermo, Palermo, Italy

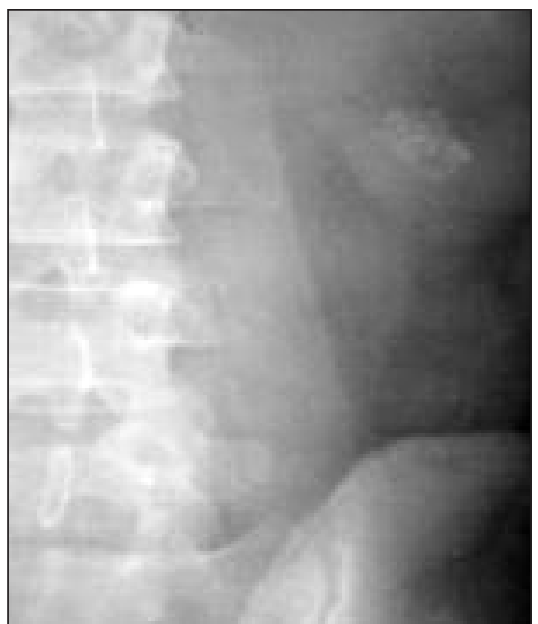

Figure 4.

\section{Discussion}

Citrates have an important role in the prevention of kidney stones because they promote formation in the urine of soluble complexes with calcium which determine a reduction of the concentration of calcium ions and of the urinary saturation of calcium oxalate and calcium phosphate.

Furthermore citrates are capable of inhibiting the crystallization, the growth and aggregation of lithogenic crystals that constitute the stone.

The magnesium potassium citrate treatment improves the clearance of residual fragments after SWL.

Recommended daily intake: ranges 40 to $60 \mathrm{mEq}$ in two/three divided doses in a continuous manner and for cycles of at least three months.

\section{REFERENCES}

1. Marangella M, Bagnis C, Bruno $M$, et al. Crystallization inhibitors in the pathophysiology and treatment of nephrolithiasis. Urol Int. 2004; 72(suppl 1):S6-10.

2. Baggio B, Borghi L, Caudarella R, et al. Linee guida per la nefrolitiasi. G Ital Nefrol. 2000; 17:59-64.

3. Fine JK, Pak CYC, Preminger GM. Effect of medical management and residual fragments on recurrent stone formation following shock-wave lithotripsy. J Urol. 1995; 153:27-33. 